\title{
Un análisis crítico del Espacio Europeo de Educación Superior como reforma de la enseñanza universitaria: los programas Verifica y Docentia en el contexto español \\ Uma análise crítica do Espaço Europeu de Educação Superior como reforma do ensino universitário: os programas Verifica \\ e Docentia no contexto espanhol
}

\section{A critical analysis of the European Higher Education Space as a university educational reform: the Verifica and Docentia programs in the Spanish context}

\author{
Juan M. Escudero Muñoz ${ }^{1}$ \\ Felipe Trillo Alonso ${ }^{2}$
}

\begin{abstract}
RESUMEN
El texto está organizado en dos apartados. El primero ofrece una perspectiva teórica que hace posible un análisis ideológico y crítico de la actual reforma universitaria española. Se argumenta que lejos de ser reductible tan solo a cuestiones organizativas y pedagógicas, representa una forma intencional de intervención estatal, y de otros agentes poderosos sobre los sistemas educativos, supeditándolos a una lógica mercantilista. En esa dirección, tomando algunos conceptos interpretativos de las aportaciones de Stephen Ball y otros
\end{abstract}

DOI: $10.1590 / 0104-4060.42112$

1 Universidad de Murcia. Facultad de Educación. Departamento de Didáctica y Organización Escolar. Murcia, España. Campus Universitario de Espinardo.30100.E-mail: jumaes@um.es

2 Universidad de Santiago de Compostela. Facultad de Ciencias de la Educación. Departamento de Didáctica y Organización Escolar. Santiago de Compostela, España. Campus Vida. 15782.E-mail: felipe.trillo@usc.es 
autores, se explica la noción de cultura performativa, que relacionamos con la obsesión por los resultados, y la desregulación, re-regulación y fabricaciones en tanto que una forma de gobierno que detrás de una aparente autonomía implica realmente una sobre-regulación que conduce al cultivo de nuevas identidades organizativas y profesionales en la universidad. En el segundo, precisada la metodología, se analizan dos programas que, en el contexto español, pueden ser considerados como un buen ejemplo de las medidas adoptadas para la garantía de la calidad educativa en el Espacio Europeo de Educación Superior. Se trata del denominado Verifica, que acredita los planes de estudio de los grados y postgrados, y el denominado Docentia, que evalúa la capacidad docente de los profesores. Sobre ambos, nos preguntamos, entre otras cuestiones: ¿En qué grado muestran características propias de la cultura performativa descrita? ¿Qué regulaciones y formas de gobierno prevalecen?

Palabras-clave: reforma de la educación universitaria; evaluación de la calidad; performatividad; regulación; fabricaciones; acreditación de titulaciones y docentes.

\section{RESUMO}

O texto está organizado em duas partes. A primeira apresenta uma perspectiva teórica para a análise ideológica e crítica da atual reforma universitária na Espanha. Argumenta-se que longe de ser uma redução a questões organizativas e pedagógicas, representa uma forma intencional de intervenção do Estado e outros agentes poderosos sobre os sistemas de ensino, sujeitando-os a uma lógica mercantilista. Neste sentido, são adotados alguns conceitos de interpretação de Stephen Ball e outros autores, tais como a cultura performativa, que relacionamos à obsessão por resultados, e os conceitos de desregulamentação, re-regulação e fabricações, que revelam uma forma de governo que, por trás de uma aparente autonomia, implica realmente um aumento da regulação, levando a novas identidades organizacionais e profissionais na universidade. Na segunda parte, uma vez descrita a metodologia, são analisados dois programas que, no contexto espanhol, podem ser considerados como um bom exemplo das medidas tomadas para garantir a qualidade da educação no Espaço do Ensino Superior Europeu. Um é o Verifica, que credencia os currículos dos cursos de graduação e de pós-graduação, e o outro é o Docentia, que avalia as competências pedagógicas dos professores. Sobre ambos, nos perguntamos, entre outras questões: Até que ponto são exemplos de uma cultura performativa? Que regulamentos e formas de governo prevalecem?

Palavras-chave: reforma educacional da universidade; avaliação da qualidade; performatividade; regulamento; fabricações; acreditação de titulações e docentes. 


\begin{abstract}
The paper is organized in two parts. A theoretical framework for the analysis of the current Spanish university reform is presented in the first part. It is an ideological and critical analysis that tries to reveal how and why the reforms, not confined to organizational and pedagogical issues, represent an intentional form of State and other powerful agencies' intervention on educational systems, which is market-oriented. In this direction, we adopt some interpretive concepts of Stephen Ball and other authors such as the concept of performativity culture related to the obsession with the results and the concepts of deregulation, re-regulation and fabrications as peculiar forms of government that seem to have more autonomy, but they actually imply a greater regulation producing new organizational and professional identities in the university. In the second part, the theoretical framework is applied in order to describe and value two programs that, in the Spanish context, can be considered as good examples of some of the decisions aiming to guarantee the quality of education in the European Higher Education Space. The programs are the called Verifica, which accredits curricula and Graduate Degrees, and the called Docentia, which evaluates the teaching skills of university teachers. On both, we ask, among other questions: To what degree are both of them singular examples of a performativity culture? What kinds of regulation and forms of government prevail?
\end{abstract}

Keywords: university educational reform; quality assessment; performativity; regulation; fabrications; career and teachers' accreditation schemes.

\title{
Introducción
}

Desde la Declaración de Bolonia (1999) hasta la fecha, el Espacio Europeo de Educación Superior representa el marco regulador de la actual reforma universitaria, especialmente en materia de enseñanza y aprendizaje. Se trata de un cambio estructural y formal pero fluido y ambiguo, uniformador y basado en una coordinación entre los países miembros, conservando cada uno espacios propios de competencia. No es extraño, entonces, que haya concitado adhesiones explícitas y controversias, acuerdos de principio y de gestión que, sin embargo, conllevan diversos frentes políticos, sociales y culturales complejos. Según algunos analistas (NÓVOA; LAWN, 2005; NÓVOA, 2010), sus objetivos ambiciosos representan promesas, dilemas y contradicciones estructurales e ideológicas, así como asuntos delicados atribuibles a su gobierno supranacional, nacional y local. 
En el Proceso de Bolonia, la docencia y el aprendizaje constituyen un ámbito más relevante y es objeto específico de este artículo. Nos vamos a ocupar de dos programas en particular, Verifica (elaboración de titulaciones) y Docentia (evaluación del profesorado). Nos proponemos analizarlo desde una determinada perspectiva teórica que ofrece dos dispositivos conceptuales pertinentes. Serán aplicados a dos Guías que la Agencia Nacional Española de Calidad (ANECA) elaboró para tales efectos.

\section{Una perspectiva teórica para el análisis y la valoración de la actual reforma universitaria española}

El Espacio Europeo de Educación Superior (EEES) puede definirse como una forma de intervención estatal, y de otros agentes sociales y económicos poderosos, sobre los sistemas escolares. Afectan a estructuras, finalidades, contenidos, metodologías didácticas y evaluación, al gobierno de las instituciones y la profesión docente y, asimismo, a las relaciones del sistema con la sociedad. De modo que los cambios no son exclusivamente pedagógicos y organizativos, sino también sociales, políticos, económicos e ideológicos. Históricamente ha sido así y, ahora, en el actual contexto de crisis no han hecho sino manifestarse con una mayor complejidad y controversias. Las reformas educativas de las últimas décadas forman parte de una suerte de "epidemia global" (BALL, 2010), que no ha surgido por azar, sino intencionalmente provocada por organismos internacionales como el Banco Mundial, el Fondo Monetario Internacional, Organización para la Cooperación y el Desarrollo Económico (OCDE) y una presencia creciente de grandes corporaciones internacionales en la educación.

Está siendo objeto de análisis y valoraciones heterogéneas. Al lado de las más posibilistas abundan otras escépticas y francamente críticas. En esencia, por su alineamiento con el orden global y hegemónico neoliberal: desbordando sus ámbitos económicos originarios, pretende calar y colonizar todos los ámbitos de la vida personal y social, la educación entre ellos. (OZGA; LAWN, 2014). De ahí que diversas voces críticas hayan acometido la tarea de desvelar y denunciar cómo el reformismo corriente va directamente dirigido a racionalizar, disciplinar y someter los sistemas escolares a la horma mercantil. (APPLE, 2002; BALL, 2010; WILLIAMSON, 2013; OZGA, 2014). 


\subsection{La cultura performativa y la obsesión por los resultados}

La noción de cultura performativa pertenece al núcleo del discurso y las políticas a través de las que se formula y proyecta sobre la educación el régimen neoliberal. El concepto de performatividad (BALL, 2002, 2003, 2010) no tiene una traducción directa en lengua castellana o portuguesa, pero una serie de términos como resultados, desempeños, exhibiciones públicas, performances permiten hacer una idea de sus significados e implicaciones.

En esencia, supone un discurso normativo que confiere un valor prevalente a las actuaciones (desempeños) y los logros (resultados, rendimientos) de los sujetos (estudiantes, docentes u otros), así como de las instituciones escolares y sus proyectos. Los estándares, las competencias y los indicadores constituyen resortes para determinar y requerir performances, así como crear sistemas de rendición de cuenta que vigilen y sancionen sus realizaciones. Pueden ser considerados como "tecnologías de regulación y control" (BALL, 2003) a través de las cuales no solo se provocan ciertos cambios estructurales sino también la construcción de identidades organizativas, docentes y estudiantiles.

Al privilegiar desempeños (performances), se enfatiza lo externo, observable, medible y cuantificable. Se deja en la sombra y se desconsidera lo interno, las meras declaraciones, lo que es más difícil de medir y cuantificar, así como los procesos, los contenidos y los contextos singulares. Los resultados (rendimientos), como también los modos de hacer estandarizados que han de ser fielmente seguidos cumplimentando procedimientos o protocolos, importan más que los contenidos, las dinámicas intermedias y los significados. Uno de los efectos más visibles de ello es la actual fiebre por tasar, indexar y cuantificar todo lo que vale: las organizaciones y las personas son reducidas a datos, a indicadores, a méritos reglados y selectivos, a comparaciones y ranking. El conocimiento, sea en la enseñanza o en la investigación, vale en tanto que externalizado y registrado, y sometido al imperio de lo útil, práctico, efectivo y rentable, es "des-socializado". (BALL, 2003; FERREIRO, 2010).

El recurso a pruebas estandarizadas de rendimiento y la pleitesía ante el principio de publicidad y trasparencia han terminado por convertirse en referentes objetivos y únicos, no solo de los aprendizajes del alumnado, sino también del valor de las performances de titulaciones, centros, docentes o investigadores. Todo ello forma parte de un nuevo "estilo de pensamiento curricular" (WILLIAMSON, 2013) que, repleto de nuevos lenguajes, criterios y prioridades del mundo de la cibernética, la neurociencia y las ciencias del aprendizaje, están desplazando del escenario los referentes y valores sociales y culturales sin los cuales el currículo y la enseñanza pierde sustancia al dejar 
fuera sus propósitos democráticos y compromisos con la igualdad, la justicia y la equidad educativa. (BALL, 2010).

El régimen y cultura performativa están construyendo y legitimando, asimismo, un espacio de juego y de poder ocupado por una nueva "clase de expertos tecnócratas" (OZGA, 2014), así como por Agencias nacionales e internacionales que se autodefinen como independientes: Agencia Nacional de Evaluación de la Calidad y Acreditación (ANECA), European Association for Quality Assurance in Higher Education (ENQA) y otras muchas. Su papel actual va, ahora, más allá de su función de "informar política", asumiendo uno mucho más influyente, encaminado a "formar políticas basadas en evidencia". Son los protagonistas por excelencia en el diseño y la aplicación de esos mecanismos de regulación, vigilancia y sanción antes mencionados. Se trata, en suma, de una de las notas de lo que la OCDE (2012) califica como el nuevo escenario de la "economía y gestión del conocimiento". Confinados los Estados a la creación de condiciones favorables para que los proveedores y clientes establezcan relaciones "libres" y recíprocas basadas en la información y la trasparencia, los expertos en estándares, indicadores, sistemas de evaluación y rendición de cuentas han conquistado espacios de poder incuestionados. Y, amparados por la cultura y las políticas de las performances, son los árbitros encargados de definir al mismo tiempo la partida de juego, las reglas, el lugar y las responsabilidades de los jugadores.

\subsection{Desregulación, re-regulación y fabricaciones, una nueva forma de gobierno}

En tanto que un régimen globalizado, la cultura en cuestión desborda, por arriba, a los gobiernos nacionales y, por abajo, acosa a las organizaciones y los profesionales con sus regulaciones, controles y sanciones. Su forma de gobierno no consiste en la tradicional intervención directa y jerárquica de los Estados. Se establece, más bien, sobre la descentralización de tareas y responsabilidades y la recentralización de objetivos, la estandarización de modos de hacer y la sanción de resultados. No se trata, por lo tanto, de forma alguna de redistribución horizontal del poder o la autonomía, sino de un modelo de gobierno paradójico con un "descontrol bien controlado". (BALL, 2010).

La presunta autonomía de las organizaciones, por lo tanto, es una delegación desde arriba y su sometimiento a criterios, estándares e indicadores de productividad y excelencia. La profesionalidad docente, una vez que fue sometida a sospecha y denostada por el discurso neoliberal, es reducida al seguimiento de directrices externas y a evaluaciones de resultados contables. Aquellas y estos han de ser trasparentes, siendo las Agencias y sus expertos lo encargados de fijar en qué han de serlo y cómo documentarlo. Es - se argumenta - algo inexcusable, precisamente por razones democráticas y de cara a satisfacer el 
derecho de una ciudadanía mejor formada y exigente de informaciones que hagan posible la "libre" elección de servicios y proveedores. (OCDE, 2012). Estamos, pues, ante un modelo singular de gobierno puesto al servicio de la conciliación de una clara lógica de ofertas y demandas. "Su generalización escribe Ball (2002, p. 13) - puede llevar a disminuir o aún destruir la capacidad de una organización para perseguir sus propósitos al verse forzada a redefinir su identidad y con ello su verdadero proyecto". De ese modo, no solo se quiere gobernar a las organizaciones educativas, sino también el alma, la vida y las prácticas de quienes en ellas trabajan, los docentes.

No está de más preguntarse, con todo, acerca de cómo las instituciones y los profesionales interpretan y reaccionan ante ello. Para referirse a ello se ha propuesto el concepto de fabricaciones. (BALL, 2010). Sus expresiones suelen ser múltiples: uso de rituales bien sonantes al declarar las propias visiones y misiones; documentación fehaciente de rutinas (performances) aplicadas a la cumplimentación de procedimientos o protocolos); establecimiento y operaciones internas de los propios mecanismos y procesos de garantía de calidad (comisiones, procesos y resultados debidamente documentados según estándares e indicadores, etc.). Dadas las regulaciones establecidas y la presión por seguirlas fielmente, es posible que las fabricaciones construidas amenacen la autenticidad de las respuestas, el sentido y la veracidad de las mismas para las instituciones y los sujetos.

Frente a los excesos de la burocracia estéril y extenuante, dichas fabricaciones pueden devenir en fachadas o simulacros, suponiendo estrategias calculadas de supervivencia. Propiciar la creación y gestión de signos y la "venta" de meras apariencias y formalidades, dañando el sentido y el valor de lo que se hace, la deliberación colegiada, genuina y contextual sobre los contenidos y la formación del alumnado. De ese modo, las reformas, con su cultura performativas y gobierno, además de provocar ciertos estructurales, podrían estar cultivando identidades organizativas y docentes simuladas, calculadoras, más atentas a lo que han de mostrar y vender hacia fuera que al desarrollo de instituciones y profesionales que busquen el sentido y la coherencia de la enseñanza y el aprendizaje universitario.

\section{Análisis y valoración del programa Verifica y Docentia}

A la luz de los dos registros analíticos que se acaban de exponer, la interpelación de esos dos programas del EEES puede abordarse procurando respon- 
der a estos interrogantes: ¿En qué grado muestran características de la cultura performativa descrita? ¿Qué elementos de la enseñanza universitaria son dichos o silenciados y a qué formas de regulación y gobierno se han sometido? ¿En qué grado las respuestas discurren por fabricaciones simuladas o por respuestas auténticas? Hemos tratado de responderlas echando mano de una metodología de análisis ideológico del discurso (KELER, 2010), aplicada a las dos Guías oficiales elaboradas y aplicadas por ANECA.

\subsection{El programa de verificación de los títulos homologados en el EEES}

Se trata del Programa regulador de los nuevos títulos cuya verificación es un requisito imprescindible para su autorización y oferta por las universidades. Se ofrece a continuación, primero, una descripción de la Guía correspondiente, segundo, una breve referencia al contexto y la historia previa para pasar, en tercer lugar, a las cuestiones que se acaban de formular.

a) La Guía para la elaboración de las titulaciones. Tal como fue diseñada por ANECA (2009), consta de diez dimensiones con elementos específicos cada una de ellas y criterios a satisfacer: 1. Descripción del título (denominación, universidad solicitante, tipo de enseñanza, número de plazas, número mínimo de créditos European Credit Transfer and Accumulation System (ECTS) a matricular por el estudiante, período lectivo e información pertinente para expedición del Suplemento Europeo del Título); 2. Justificación (interés científico, académico y profesional, referentes externos y procedimientos internos de consulta tomados en consideración al elaborarlo); 3. Objetivos (competencias generales y específicas); 4. Acceso y admisión de estudiantes (información previa, acceso y admisión, apoyo y orientación durante los estudios, transferencia y reconocimiento de créditos); 5. Planificación de la enseñanza (estructura, movilidad del estudiante, descripción de módulos o materias); 6. Personal académico (profesorado y otros recursos disponibles, adecuación al plan de estudios); 7. Recursos materiales y servicios (medios materiales y servicios disponibles y previstos); 8. Resultados previstos (estimación de indicadores en graduación, tasas de abandono y eficiencia, procedimientos para valorar el proceso y los resultados del aprendizaje); 9. Sistema de garantía de calidad (responsabilidades, procedimientos de evaluación y mejora de la calidad de la enseñanza y el profesorado, para garantizar la de las prácticas externas y analizar la inserción laboral y su satisfacción con la formación, así como la de otros colectivos, previsiones respecto a la posible extinción del título); 10. Calendario de implantación (cronograma, adaptación de los estudiantes procedentes de los planes de estudio previos, enseñanzas que se extinguen). 
De acuerdo con tal regulación, el proceso quedó bien pautado y secuenciado (TRILLO, 2005): elaboración de las titulaciones por las universidades, inscripción y remisión a ANECA para su evaluación, emisión por parte de la agencia de un informe preceptivo con una sanción positiva favorable, verificación, o con requerimiento adicionales a subsanar, debiendo cubrir de nuevo los pasos correspondientes. Verificada una titulación y autorizada por las autoridades competentes, cada universidad estaba en condiciones formales de ofertarla.

b) Historia previa y contexto. El Proceso de Bolonia representó, en esta materia, un antes y un después en el contexto nacional. Pero, como otras reformas, el EEES no cayó sobre el vacío. Desde los ochenta fueron sucediéndose otras reformas de los planes de estudio con cuyo legado entró en relación el tema que nos ocupa. Un legado, por cierto, cuyas lecciones debieran haberse tenido bastante más en cuenta. Rodríguez y otros (2006), por citar un caso, habían señalado que las reformas precedentes fueron más administrativas, estructurales y organizativas y, mucho menos, capaces de renovar los contenidos, las metodologías, los aprendizajes discentes y la evaluación de los mismos. Asimismo, dicen, la autonomía formal decretada y conferida a las universidades, departamentos y comisiones fue ambivalente. La sobrerregulación de los estudios por el Ministerio y, además, la micro-política entre áreas de conocimiento y departamentos la vaciaron de contenidos sustantivos en algunos casos y, en otros, la hicieron prisionera de la correlación de fuerzas e intereses particulares. (ESCUDERO, 2012). Con tales residuos, que no fueron básicamente alterados, se encontró la tarea de redefinir las nuevas titulaciones.

c) Un análisis cauteloso de las preguntas exploratorias planteadas. Tomando en consideración las cuestiones planteadas, la Guía que se analiza responde bien a las características descritas en el marco teórico dispuesto.

Una fuerte regulación desde arriba. A nuestro entender, las diez dimensiones referidas y, todavía más, los más de ochenta indicadores que habían de ser cumplimentados y satisfechos, son la muestra fehaciente de un afán tan regulador que llegó hasta los más ínfimos detalles. Al mismo tiempo, son la expresión de que los reformadores y expertos albergaban tanto una gran convicción de la bondad de los cambios pretendidos, como una desconfianza respecto a la voluntad y la capacidad de las universidades y los docentes para acometer la reforma planteada. Un acreditado balance (TARRACH, 2011, p. 8) ha escrito que "[...] una de las principales amenazas del proceso consistió en regularlo a golpe de decreto" e que "[...] el sistema actual de acreditación de programas muestra un alto grado de controles de calidad extenuantes que no aportan beneficios claros en términos de mejora". (TARRACH, 2011, p. 16). La regulación y las exigencias de performances, en la versión nacional del EEES y en el tema que nos ha ocupa, ha dado patentes de la fiebre que estamos denunciando. 
Un afán irrelevante y obsesivo en reclamar datos, indicadores y previsiones de cada titulación, los indicadores referidos, por ejemplo a las tasas previsibles de abandono y de empleabilidad, son una muestra. Sin entrar en una discusión sobre su razonabilidad, llegaron a ser fabricaciones inducidas por las regulaciones externas pero inauténticas, instrumentales para lograr la verificación pero no veraces.

Un discurso regulador centrado en determinados elementos del diseño (objetivos, competencias, indicadores de rendimiento o performances) a costa de otros como los contenidos. De hecho, entre los diferentes modelos posibles para el diseño de la formación, la Guía de ANECA optó sin justificación por modelo de objetivos (medios-fines), dejando ostentosamente fuera el modelo alternativo de proceso. (ESCUDERO, 2012). No solo se apostó por conferir un peso destacado a las competencias (listas sin fin ni concierto de performances), sino que otros elementos, en concreto los contenidos, se definieron como instrumentales respecto a aquellas: era preciso un cambio de paradigma desde la enseñanza al aprendizaje. El lenguaje que presidió el proceso se inspiró más en la cultura gerencial que en la educativa. Ello justificó que el informe Aarhus Declaration (2011) censurara ausencias flagrantes sobre cuestiones de equidad, estatus socioeconómico, género y origen étnico del alumnado, así como otras relativas al abandono, las rutas de acceso a los estudios o el reconocimiento de los aprendizajes previos.

$\mathrm{Al}$ modelo de gobierno, por su parte, le cuadra bien una valoración del mismo como desregulador y re-regulador simultáneamente. En un sentido, porque la fuerte regulación por parte de ANECA supuso un caldo de cultivo propicio a fabricaciones simulacro. Con el tiempo ha ido afianzándose en las instituciones y el profesorado la máxima de "hagamos lo que se nos exige para salir del paso y ya entraremos posteriormente, si viene al caso, en lo importante". En otro, porque la elaboración de las nuevas titulaciones no logró superar, sino más bien reiterar, aquellos retos del pasado reformista que seguían pendientes. La micro-política y la correlación de fuerzas locales ha seguido presidiendo tanto las decisiones formales como las sustantivas: una forma curiosa de aplicación de la lógica mercantil que, desde luego, también puede ser interna.

En resumidas cuentas, la versión española del EEES, en materia de rediseño de la formación, es una buena muestra de los supuestos, prácticas y consecuencias de la cultura perfomativa y de ese modelo de gobierno con una aparente autonomía pero fuertemente controlada. 


\subsection{El programa Docentia ¿qué aseguramiento de la calidad docente?}

Docentia es la denominación empleada para referirse al Programa de Apoyo a la Evaluación de la Actividad Docente del Profesorado Universitario, desarrollado por ANECA. Concebido a finales del año 2006, se dio a conocer a las diversas Agencias Autonómicas en febrero de 2007. Su objetivo declarado es "[...] apoyar a las universidades en el diseño de mecanismo propios para gestionar la calidad de la actividad docente del profesorado universitario y favorecer su desarrollo y reconocimiento". (<http://www.aneca.es/Programas/ DOCENTIA $>$ ). Su legitimidad deviene del organismo que lo propone, ANECA: una fundación estatal, creada por Acuerdo del Consejo de Ministros el 19 de julio de 2002 en cumplimiento de lo establecido en la Ley Orgánica de Universidades (6/2001 de 21 de diciembre, LOU), que tiene como objetivo "[...] contribuir a la mejora de la calidad del sistema de educación superior mediante la evaluación, certificación y acreditación de enseñanzas, profesorado e instituciones."

En nuestro análisis del Programa Docentia seguiremos la misma estructura y orientación que la adoptada en el Programa Verifica.

a) La concepción y el desarrollo del Programa. No hay un único documento susceptible de análisis al que podamos acudir para resumir lo esencial del Docentia. Todo, en conjunto, bastante prolijo.

En una primera y rápida visión cabe decir lo siguiente sobre el Docentia: como objetivo general sirve el declarado al inicio. El marco de las relaciones entre las universidades y ANECA se define así:

En el actual ordenamiento del sistema universitario español la garantía de la capacitación y competencia del profesorado descansa en las universidades. Del mismo modo, son las administraciones públicas las que, a través de las agencias de evaluación aseguran el cumplimiento de unos estándares básicos. (ver: $<$ http://www.aneca.es $>$ ).

Desde esa perspectiva, se auto-titula como un proceso de evaluación interno en el que participan agentes externos. Cabe añadir que los efectos de este Programa se hacen sentir directamente sobre la política de profesorado de las universidades en aspectos tales como la formación y desarrollo (por la vía de la reflexión del profesor), la promoción personal y profesional o los incentivos económicos (complementos retributivos al profesorado).

Una lectura más detenida del Programa Docentia destaca los siguientes aspectos: que presenta un definición de la actividad docente que se ocupa de 
la desarrollada tanto en el aula como fuera de ella; que presenta también una definición de evaluación de la actividad docente considerando su rol profesional y su contribución para conseguir los objetivos de la titulación en la que está implicado; que las dimensiones a evaluar son la planificación, el desarrollo y los resultados de la enseñanza; que las fuentes y los procedimientos de evaluación son el auto-informe del profesor, el informe de los responsables académicos y la encuesta a los estudiantes. Por fin, que todo ello se hace conforme a los siguientes criterios de evaluación de la actividad docente: adecuación, que implica que responde a los requerimientos establecidos por la universidad y el centro; satisfacción, que conlleva generar una opinión favorable de los demás agentes implicados en el proceso, en especial de estudiantes, colegas y responsables académicos; eficiencia, que estima el logro en los estudiantes de los objetivos previstos; y orientación a la innovación docente, entendida como una reflexión del profesor sobre su propia práctica que favorezca su aprendizaje.

Por último, un análisis algo más pormenorizado pone de manifiesto lo siguiente: En primer lugar, la existencia de hasta cuatro comisiones distintas para afrontar este Programa, con la consiguiente exigencia de proceder a su configuración mediante la selección y preparación ad hoc de sus miembros. En segundo lugar, que el objeto de evaluación varía según las comisiones, una se ocupa de evaluar a los profesores, otra de evaluar el diseño para evaluar a los profesores, otra de evaluar el proceso mientras se lleva a cabo, y otra de evaluar el informe final. En tercer lugar, que cada una de ellas cuenta con Guías específicas para orientar y facilitar su trabajo, en las que se proporcionan frases que identifican actividades docentes concretas así como expresiones valorativas para pronunciarse acerca de los mismos. Y por último, que sin salirse del guión que todo eso representa, cada universidad elabora su propio modelo, procedimiento, guía y herramientas, especificando en algunos casos hasta el detalle las actividades docentes susceptibles de ser evaluadas y la consiguiente cuantificación que merezcan.

b) El Programa Docentia y la evaluación del profesorado, contexto e historia. El propio documento que presenta el Modelo del Programa Docentia hace una breve descripción del estado de la evaluación del profesorado universitario en España desde la década de los 1980. Advierte que esa actividad no había alcanzado la sistematicidad y el rigor que algunos demandaban. Según esto, podríamos decir que Docentia llega a la universidad en un tiempo en el que, para la mayoría y por diversas razones, la evaluación del profesorado no goza de buena reputación. El resurgir del tema con el Programa Docentia, en consecuencia, se recibe de manera muy dispar, pero no con indiferencia, y las reacciones van desde el escepticismo hasta la hostilidad pasando por el consentimiento y, en 
un menor grado, la complicidad. Entusiasmo, ninguno. Sólo el axioma de la incorporación al EEES le proporciona alguna credibilidad. (POZO et al., 2011).

c) Un análisis cauteloso de las preguntas exploratorias planteadas. Sobre el Programa Docentia afirmamos sin ambages que también es posible calificarlo como una tecnología performativa de control. Veamos los argumentos, utilizando para ello los mismos epígrafes que en el caso del Verifica al objeto de facilitar el contraste entre ambos programas.

Una fuerte regulación desde arriba del diseño de los planes de evaluación de la actividad docente que deben desarrollar las universidades. Pese a la declaración inicial del Docentia de servir para "apoyar" a las universidades, lo que en realidad acontece es que impone en la práctica una manera casi única de proceder. La inquietud por no obtener la certificación, necesaria por obligada para acreditarse, deja escaso margen de autonomía de las universidades. Según esto, se hace evidente que la competencia de las universidades de garantizar la capacitación de su profesorado es una tarea que tiene que ser tutelada por las administraciones públicas a través de las agencias de evaluación. Según esto, se pone de manifiesto una clara desconfianza de la administración respecto a la universidad a la que, según el ordenamiento actual, hay que ponerle un tutor que vigile todas sus realizaciones.

Un afán bastante irrelevante e inefectivo por reclamar datos y determinados indicadores, que se revelan como "respuestas fabricadas" más orientadas a conseguir la certificación que se solicita, que como garantía de que lo que se presenta responde no sólo a la realidad de los hechos sino también a lo que éstos pueden tener de más "auténtico".

Veamos en qué medida lo que se hace atiende más a aspectos burocráticos: a nivel de planificación, todo puede quedar en una mera relación de asignaturas impartidas, guías docentes presentadas, objetivos declarados, condiciones de tiempo y espacio genéricas, recursos generales, pero sin justificar desde un punto de vista teórico y defendible los criterios didácticos empleados para su adopción. A nivel de desarrollo puede acontecer otro tanto, aspectos como el porcentaje de asistencia al aula de los estudiantes, o la asistencia del profesor a los órganos colegiados, el cumplimiento por éste del horario y de las fechas establecidas, su utilización de las Tecnologías de la Información y la Comunicación (TIC) para facilitar materiales y mantener una comunicación con los estudiantes, o incluso la lengua vehicular utilizada en el aula (en el caso de las universidades pertenecientes a comunidades bilingües), son el tipo de datos medibles que centran la atención - que poco dicen respecto de la adecuada y razonable selección de contenidos, del interés de las tareas, del favorable clima de aula, de la coherencia y justicia en la evaluación. Finalmente, a nivel de resultados es evidente que el número de estudiante matriculados, las tasas de éxito, 
las convocatorias de evaluación que precisan, sus declaraciones de satisfacción junto con las de los profesores sobre apenas unos descriptores generales, o en otro orden la asistencia de los profesores a cursos de formación, son datos que no proporcionan una información relevante sobre la calidad de los procesos de aprendizaje desarrollados por los estudiantes (no explican si la han comprendido o solo reproducido de memoria) y tampoco aclaran los procedimientos de evaluación utilizados (¿exámenes con tareas de memoria o rutina o proyectos para resolver problemas complejos?); asimismo, su satisfacción puede devenir de la consecución de un aprobado fácil y, desde luego, que los profesores asistan a cursos de formación no implica que automáticamente lo apliquen en el aula (ni que lo aprendido merezca serlo).

Pero hay más, pues en el marco del Programa Docentia puede ocurrir (sucede de hecho) que en algunas universidades los resultados puntúan más que la planificación, o que en el desarrollo de la actividad docente la presentación en tiempo de una guía docente en una plataforma virtual se puntúe más que promover un clima de mutua interrogación y diálogo en el aula sobre un contenido relevante. Siendo así estaríamos ante un inequívoco ejemplo de lo que Ball llama "fabricaciones" que desvirtúan el acceso a una información "auténtica".

Un discurso regulador con un énfasis en determinados elementos formales de la actividad docente (la cumplimentación de formularios, la elaboración de guías, la utilización de plataformas virtuales, la coordinación de prácticas y de programas de intercambio para estudiantes, etc.) y la desconsideración de otros más "auténticos", como lo supone el asumir una responsabilidad formativa con los estudiantes, y en concreto, a través del desarrollo de las competencias, que no se evalúan, que no están presentes o no al menos de la manera destacada que le correspondería a la vista de lo que representan para el diseño de los planes de estudio.

El modelo de gobierno que subyace al Programa Docentia es una buena muestra de la desregulación re-regulada. Como vimos, Ball (2003) denuncia una forma de ejercer el gobierno que, mediante evaluaciones, informes y retribución por desempeño, dejan a la mayoría de los subalternos "expuestos" a los mecanismos de control. Tal cual lo que hace el Docentia. Pero lo más extraordinario de esta forma de gobernar es que, para imponerse, no necesita apenas ejercer la violencia, porque lo que se consigue es "inculcar performatividad en el alma del trabajador". (BALL, 2003, p. 90). Pensando en los profesores, sostiene que, seducidos por los discursos sobre la excelencia y el éxito, “[ [... caen en la performatividad debido a la diligencia con que intentan cumplir los nuevos imperativos de la competición y el logro de objetivos" (BALL, 2003, p. 92), que interiorizan, por lo tanto, una racionalidad técnica, más preocupada por cómo hacer lo que les dicen que hagan, que por reflexionar y posicionarse al 
respecto. Pues bien, un buen ejemplo de esto podemos encontrarlo en los auto-informes del Programa Docentia, en los que los profesores se ven impelidos a "fabricar" una imagen de sí mismos que se ajuste al modelo: haciendo lo que sea por sobresalir o por sobrevivir. Es la primacía de la "razón instrumental". Que ignora la necesidad de un consenso, previo debate, para definir algo tan complejo e incierto como es lo que entendemos por ser un buen profesor. (DE MIGUEL, 1998; ZABALZA, 2008).

En definitiva, nuestra aproximación al Programa Docentia como una vía de análisis del EEES nos lleva a la misma conclusión alcanzada respecto al Verifica. Visto lo visto del Docentia, es obvio que conduce a una concepción del desempeño profesional que se caracteriza por el cumplimiento de una serie de reglas generadas e impuestas desde fuera. Según esto, responder al Docentia representa un ejemplo más de evaluación meramente orientada a la rendición de cuentas con propósitos sumativos (acreditación) y una forma de control amenazante pero sin efectos realmente formativos.

En resumidas cuentas, los dos programas analizados desde la perspectiva teórica empleada ofrecen ciertas evidencias de que el EEES no ha quedado libre de los afanes y los efectos de la cultura performativa dominante ni, tampoco, de un modelo con tan fuertes regulaciones que es apto para cercenar los márgenes razonables de autonomía y autenticidad pedagógica.

La fiebre por los procedimientos formales desconoce flagrantemente la complejidad y la fundamentación relevante que requieren las decisiones sobre los contenidos de la formación, así como todos los demás elementos de la enseñanza y aprendizaje. La regulación procedimental de los procesos, sean referidos al diseño de los estudios o a la enseñanza, restringe e incluso puede anular las tareas interpretativas, reflexivas, críticas y situadas de las instituciones y el profesorado. Con ello no solo se estaría poniendo en cuestión la relevancia y el rigor cultural de la enseñanza, sino también las dinámicas y las relaciones colegiadas que son precisas para construir y comprometer proyectos organizativos y pedagógicos coherentes, sólidos, debidamente concertados y llevados a cabo.

En relación con el modelo de gobierno subyacente y activado para gestionar el Proceso de Bolonia, caben dudas razonables acerca de cuál podría ser, en último extremo, su agenda no confesada. Llama poderosamente la atención el hecho de que los reformadores y expertos hayan podido ser tan inconscientes respecto a un saber bien establecido respecto a los cambios educativos. El abuso de sobrerregulaciones externas, no solo es un recurso estéril para transformar las culturas y las prácticas, sino una política que puede llevar a lo contrario de lo que dice pretender. En efecto, favorece que las instituciones y los sujetos recurran a fabricaciones estratégicas para lograr sanciones positivas, pero no relevantes y efectivas para repensar y mejorar la enseñanza y el aprendizaje universitario. 
La metodología que hemos utilizado para ofrecer nuestra mirada sobre el EEES tiene, desde luego, sus limitaciones: nos hemos fijado más en textos y discursos que en prácticas concretas. No queremos sostener en ningún modo que haya habido centros y docentes que, al socaire de Bolonia, hayan comprometido y desarrollados ideas y prácticas valiosas. Sin duda ninguna los ha habido. Así y todo, las referencias hechas al diseño desde arriba del Proceso de Bolonia debiera llevar a una revisión detenida de qué es lo que se sigue pidiendo de los centros y qué es lo que se espera que hagan los docentes al querer renovar y mejora su desarrollo profesional y la formación del alumnado.

\section{REFERENCIAS}

AARHUS DECLARATION. Investing today in talent tomorrow. 2011. Disponible en: $<$ http://www.eua.be/Libraries/Newsletter/Aarhus_Declaration.sflb.ashx $>$. Acceso en: 20 mar. 2014.

ANECA. Guía de apoyo para la elaboración de memoria para la solicitud de verificación de títulos oficiales (grado y máster). 2009.

APPLE, M. Educar como dios manda. Barcelona: Paidós, 2002.

BALL, S. Reformar escuelas, reformar profesores y los terrores de la performatividad. Revista Portuguesa de Educação, v. 15, n. 2, p. 3-33, 2002.

BALL, S. Profesionalismo, gerencialismo y peformatividad. Revista de Educación y Pedagogía, v. 15, n. 36, p. 87-104, 2003.

BALL, S. Performatividad y fabricaciones en la economía educacional: en pos de la sociedad performativa. Educaçao \& Realidade, v. 35, n. 2, p. 37-55, 2010.

DE MIGUEL DÍAZ, M. La evaluación de la función docente del profesorado universitario. En: RODRÍGUEZ, J. M. Seminario sobre formación y evaluación del profesorado universitario. Huelva: Ice Universidad de Huelva, 1998. p. 123-138.

ESCUDERO, J. M. La elaboración de las titulaciones universitarias en el contexto del EEES: condiciones, procesos, resultados e interrogantes. En: BAUTISTA MARTÍNEZ, J. M. Innovación en la Universidad: prácticas, políticas y retóricas. Barcelona: Graó, 2012. p. 53-84.

FERREIRO, X. Mercantilización y precarización del conocimiento. En: VERCELLONE, C. La universidad en conflicto: capturas y fugas en el mercado global del saber. Madrid: Traficantes de Sueños, 2010. p. 113-142. 
KELLER, R El análisis del discurso basado en la sociología del conocimiento: Un programa de investigación para el análisis de relaciones sociales y políticas de conocimiento. Forum: Qualitative Social Research, v. 11, n. 3, 2010.

NÓVOA, A. La construcción de un Espacio Europeo de Educación: gobernando a través de los datos y la comparación. Revista Española de Educación Comparada, v. 16, p. 23-41, 2010.

NÓVOA, A.; LAWN, M. L'Europe reinventée: regard critique sur l'espace européen de l'éducation. Paris: L'Harmattan, 2005.

OECD. Exploring the complex interaction between governance and knowledge in education. Paris: OECD, 2012.

OZGA, L. Outsourcing the governing of education: the contemporary inspection of schooling in England. Sisyphus: Journal of Education, v. 2, n. 1, p. 89-105, 2014.

OZGA, J.; LAWN, M. Framework of regulations: evidence, knowledge and judgement of inspection. Sisyphus: Journal of Education, v. 2, n. 1, p. 7-15, 2014.

POZO, C.; BRETONES, M.; MARTOS, J.; ALONSO, E. Evaluación de la actividad docente en el Espacio Europeo de Educación Superior: un estudio comparativo de indicadores de calidad en universidades europeas. Revista española de pedagogía, v. 248, p. 145-163, 2011.

RODRÍGUEZ, C.; GÁMEZ, R.; ÁLVARES, J. Caracterización de los desajustes asociados a la reforma de los planes de estudio universitarios en España: el caso de la Facultad de Ciencias de la Educación de la Universidad de Granada. Profesorado: Revista de Currículum y Formación del Profesorado, v. 10, n. 2, p. 1-13, 2006.

TARRACH, R. Audacia para llegar lejos: universidades fuertes para la España del mañana. Informe. 2011. Disponible en: <http://www.usc.es/export/sites/default/gl/web/ descargas/informe-finaleu2015.pdf $>$. Acceso en: 20 mar. 2014.

TRILLO, F. El Espacio Europeo de Educación Superior: dilemas, incertidumbres y perversiones. 2005. Disponible en: <http://firgoa.usc.es/drupal/node/21486>. Acceso en: 20 mar. 2014.

WILLIAMSON, B. The future of the curriculum: school knowledge in the digital age. MacArthur Foundation, 2013. Disponible en: <www.macfound.org > Acceso en: 20 mar. 2014.

ZABALZA, M. A. El Espacio Europeo de Educación Superior: innovación en la enseñanza universitaria. Innovación Educativa, n. 18, p. 69-95, 2008.

Texto recebido em 13 de julho de 2015. Texto aprovado em 17 de agosto de 2015. 
